\title{
0 Clostridium difficile como agente indutor de diarréia inflamatória
}

\author{
Clostridium difficile as an inflammatory diarrhea inducer agent
}

\author{
Marcos Fábio G. Rocha, José Júlio C. Sidrim e Aldo Ângelo M. Lima
}

\begin{abstract}
Resumo O Clostridium difficile tem sido apontado como um importante agente causador de doenças diarreicas associadas ao uso de antimicrobianos. Contudo, em razão da sua complexidade a fisiopatologia dessas doenças ainda se encontra apenas parcialmente esclarecida, muito embora, uma série de trabalhos científicos demonstrem a importância das toxinas $A$ e $B$ na patogênese da diarréia inflamatória induzida por esse microrganismo. Os mecanismos inflamatórios envolvidos nas atividades biológicas dessas toxinas são bastante complexos. Existem alguns estudos relatando que a toxina $B$ é desprovida de efeitos enterotóxicos, in vivo. No entanto, essa toxina provoca, de forma dose-dependente, alterações eletrofisiológicas e morfológicas na mucosa colônica humana, in vitro. Ademais, a toxina $B$ estimula a síntese de potentes mediadores inflamatórios, por monócitos e macrófagos. Os efeitos provocados pela toxina $A$ sobre a mucosa intestinal são bastante evidentes $e$ caracterizam-se por uma intensa secreção de fluidos e por um grande acúmulo de células inflamatórias, do tipo macrófagos, mastócitos, linfócitos e neutrófilos, com a conseqüente liberação de seus mediadores, tais como prostaglandinas, leucotrienos, fator de agregação plaquetária, óxido nítrico e citocinas.
\end{abstract}

Palavras-chaves: Clostridium difficile. Toxinas A e B. Diarréia. Inflamação.

\begin{abstract}
Clostridium difficile has been pointed out as an important agent of diarrheal diseases associated with antibiotic use. However, due to its complexity, the physiopathology of these diseases is only partially elucidated, although a series of scientific works has demonstrated the importance of toxins $A$ and $B$ in the pathogenesis of the inflammatory diarrhea induced by this microorganism. The inflammatory mechanisms involved in the biological activities of these toxins are complex. There are some studies demonstrating that toxin $B$ has no enterotoxic activity in vivo. However, this toxin causes dose-dependent eletrophysiologic and morphologic modifications of human colonic mucosa in vitro. In addition, toxin B stimulates the synthesis of potent inflammatory mediators by monocytes and macrophages. The effects provoked by toxin $A$ on the intestinal mucosa are quite evident and are characterized by intense fluid secretion and by inflammatory cell accumulation, such as macrophages, mast cells, lymphocytes and neutrophils, with the consequent release of mediators such as prostaglandins, leukotrienes, platelet activating factor, nitric oxide and cytokines.
\end{abstract}

Key-words: Clostridium difficile. Toxins $A$ and B. Diarrhea. Inflammation.

\footnotetext{
Departamento de Medicina Veterinária da Faculdade de Veterinária da Universidade Estadual do Ceará. Laboratório de Micologia Médica do Depto de Patologia e Medicina Legal, Unidade de Pesquisas Clínicas e Depto de Fisiologia e Farmacologia da Faculdade de Medicina da Universidade Federal do Ceará, Fortaleza, CE.

Endereço para correspondência: Dr. José Júlio C. Sidrim. Laboratório de Micologia Médica, Deptº de Patologia e Medicina Legal/UFC. R. Monsenhor Furtado s/no; Rodolfo Teófilo. Caixa Postal 3163, 60441-750, Fortaleza, CE, Brasil.

Tel: 5585 243-9301; Fax 5585 295-1736

Recebido para publicação em 15/07/98.
} 


\section{ABORDAGEM HISTÓRICA}

Uma das primeiras descrições da colite pseudomembranosa foi feita por Finney, em 1893, a partir da observação de uma pseudomembrana, presente no cólon de uma paciente que apresentou uma colite fatal depois de uma cirurgia. Contudo, vale salientar que, o agente causador desta enfermidade não foi elucidado nesta época26.

Somente em 1935, Hall e Toole, estudando a flora intestinal de recém-nascidos saudáveis, descreveram um microrganismo, até então desconhecido, que recebeu inicialmente a denominação de Bacillus difficilis e logo em seguida de Clostridium difficile, refletindo exatamente a dificuldade encontrada para isolar e manter esse microrganismo em cultura pura. Nesse mesmo ano, Hall e Toole demonstraram, ainda, que o $C$. difficile era extremamente toxicogênico em cultura. Essa evidência foi baseada no achado de lesões, parada respiratória e morte quando da injeção de cultura líquida ou filtrado do meio que continha esse microrganismo, em animais experimentais26.

Depois de sua descoberta, o Clostridium difficile passou por uma relativa obscuridade, haja visto que a colite pseudomembranosa foi uma condição rara na era pré-antibiótica. Contudo, em meados dos anos 1970 e início da década de 1980, essa enfermidade atingiu proporções epidêmicas, tornando-se uma complicação comum ao uso de antibióticos 2126.

Somente por volta de 1970, as bactérias anaeróbias começaram a ser implicadas como importantes agentes causadores de doenças. Por conseguinte, surgiram vários estudos em busca de antimicrobianos ativos contra esses agentes infecciosos emergentes; tendo sido a lincomicina um dos primeiros sucessos 21 26.

Entretanto, logo foi observado que muitos dos pacientes tratados com a lincomicina e seus congêneres freqüentemente apresentavam diarréia e uma severa inflamação da mucosa colônica. Dessa forma, esse antibiótico mostravase eficaz não somente contra as bactérias anaeróbias, mas também como um fator desencadeante da colite pseudomembranosa, possivelmente favorecendo a ativação do C. difficile que se encontrava inócuo apenas como um microrganismo da microbiota intestinal26.

A partir do final da década de setenta, o C. difficile tem sido reconhecido com freqüência cada vez maior como um importante enteropatógeno. Nesse sentido, dados epidemiológicos, nos EUA, indicam que até $1 / 4$ dos pacientes hospitalizados albergam esse agente; e que muitos desenvolvem sintomas de diarréia e colite 353842 . Corroborando este fato, Lima et al, 1993, demonstraram, em estudo realizado no Hospital Walter Cantídio da Universidade Federal do Ceará, Brasil, que o $C$. difficile é o principal agente causador de diarréia nosocomial nesse estabelecimento23.

Nos dias de hoje, há numerosos estudos laboratoriais e clínicos demonstrando que o C. difficile é o principal agente causador de doenças intestinais associadas ao uso de antimicrobianos, que variam desde diarréia autolimitada, relativamente benigna, a colite pseudomembranosa grave, que, muitas vezes, pode ser fatal21 264042 .

É importante ressaltar o fato de que, com o advento do uso indiscriminado de antimicrobianos, as doenças relacionadas ao $C$. difficile têm se tornado cada vez mais freqüentes e, dessa maneira, têm aumentado os índices de mortalidade por doenças diarréicas causadas por esse microrganismo2 621.

\section{MICROBIOLOGIA}

O C. difficile é reconhecido como um bacilo anaeróbio obrigatório, gram-positivo e com esporo subterminal, que faz parte da microbiota intestinal, tanto do homem como de outros animais. Esse agente produz duas toxinas denominadas toxina $A$ (enterotoxina) e toxina $B$ (citotoxina). Contudo, foram isoladas, em portadores humanos e animais, cepas que não produzem toxinas21 2635 .

Até por volta de 1977, era difícil se fazer a distinção entre o Clostridium difficile e outros microrganismos comumente encontrados nas fezes, como, por exemplo, o Clostridium sporogenes. Por conseguinte, as doenças associadas ao C. difficile não eram adequadamente diagnosticadas. Entretanto, George et al, 197816, desenvolveram um meio de cultura seletivo contendo cicloserina e cefoxitima e frutose em ágar, o qual facilitou sensivelmente $o$ isolamento desse agente. Vale salientar que atualmente vários sistemas de automação estão sendo utilizados para identificação de bactérias anaeróbias, incluindo o Clostridium difficile.

Em adição, existem alguns métodos laboratoriais, do tipo ELISA, cultura de células, 
contraimunoeletroforese e aglutinação pelo látex, para detecção das toxinas do $C$. difficile. Dentre esses, o ensaio utilizando a cultura de células é considerado o método padrão e, por conseguinte, serve de parâmetro para os outros testes 26 .

\section{TOXINAS}

Clostridium difficile produz duas toxinas denominadas de toxina $A$ (enterotoxina) e toxina $B$ (citotoxina), que possuem uma seqüência de 2710 e 2366 aminoácidos e pesos moleculares de 308 e $279 \mathrm{kDa}$, respectivamente 5 10. Dentre os aminoácidos que compõem essas toxinas, a aspargina, glicina e glutamina são os que se apresentam em maiores quantidades 4510 .

O modo de ação biológica dessas toxinas ainda é pouco compreendido, não obstante muitas publicações confirmarem sua participação efetiva, na patogênese de doenças diarréicas associadas a antibióticos. Tanto é verdade, que cepas de $C$. difficile que não produzem toxinas são incapazes de desenvolver as doenças diarréicas relacionadas com este microrganismo, tanto no homem como em animais experimentais3 6212635 .

Ademais, tem sido relatado que é possível um amplo espectro de resposta do hospedeiro ao $C$. difficile, passando do estado de portador assintomático até a colite pseudomembranosa. Esta variação, ainda, não é bem compreendida e pode estar associada com diferenças entre as cepas do microrganismo, ou com variações na resposta do hospedeiro, relacionadas com modificações nos receptores das toxinas ou nas defesas imunológicas 2135.

\section{DOENÇAS RELACIONADAS}

A diarréia associada a antibióticos e/ou a colite pseudomembranosa, tanto em animais como no homem, tem sido comumente relacionada ao Clostridium difficile toxigênico numa freqüência de 50 a $80 \%$, para diarréia associada a antibióticos, e 90 a $100 \%$ para colite pseudomembranosa6 1621 .

A flora intestinal, tanto do homem como dos animais, quando íntegra, impede eficazmente a colonização pelo C. difficile. Contudo, os antibióticos constituem o principal fator de alteração da microbiota bacteriana, provocando a sua substituição por microrganismos resistentes às drogas em uso, os quais proliferam e podem provocar quadros patológicos diversos.
O desequilíbrio da microbiota intestinal se manifesta especialmente com o surgimento de diarréia, ou até mesmo com um quadro grave de colite pseudomembranosa provocada pelo Clostridium difficile 213540 .

Em geral, esse quadro clínico produzido pelo $C$. difficile ocorre em pacientes que tiveram a sua flora intestinal anaeróbia alterada por antibióticos, do tipo clindamicina, ampicilina, amoxicilina, macrolídeos, lincosamidas, aminoglicosídios, cefalosporinas etc21 35 .

A colite pseudomembranosa caracteriza-se por uma severa inflamação da mucosa colônica e pela formação de uma pseudomembrana composta de fibrina, muco, células epiteliais necrosadas e leucócitos 1618 29. Esta enfermidade é diagnosticada pela observação endoscópica da pseudomembrana ou pela presença de microabcessos no cólon de pacientes com diarréia, e com história de exposição a antibiótico/quimioterápicos, nas últimas 6-8 semanas 1821.

Outra forma de manifestação da infecção pelo Clostridium difficile é o surgimento de diarréia leve a moderada, denominada diarréia associada a antibióticos. Em geral, esse distúrbio é menos grave e de tratamento mais fácil do que a forma clássica de colite pseudomembranosa21 2635.

\section{ATIVIDADES BIOLÓGICAS DAS TOXINAS A E B}

Nos últimos anos, vários pesquisadores têm se empenhado na investigação dos mecanismos moleculares, envolvendo as toxinas $A$ e $B$ do Clostridium difficile. Por conseguinte, surgiram vários trabalhos evidenciando o fato de que ambas as toxinas são capazes de monoglicosilar proteínas Rho, pertencentes à família de GTPases de baixo peso molecular9 17192039 .

O mecanismo pelo qual a glicosilação de proteínas $R$ ho provoca inativação molecular ainda não foi bem esclarecido. Entretanto, sabe-se que esse efeito se encontra relacionado, pelo menos em parte, com alterações na transdução de sinal19 39. Vale salientar que essa glicosilação de proteínas $R h o$, além de inativar várias atividades biológicas de determinadas moléculas, também é capaz de intermediar alguns eventos celulares produzidos por toxinas bacterianas 1 . Ratificando essa evidência foi demonstrado que os mecanismos de apoptose, alterações no citoesqueleto e inibição da fosfolipase $D$, provocados pelas toxinas $A$ e $B$ são dependentes 
da inativação de proteínas Rho17 192039 .

As toxinas do $C$. difficile, especialmente a toxina B, causam arredondamento celular e marginalização do núcleo. Essas mudanças morfológicas, geralmente, são irreversíveis e inibem a divisão celular. Dessa maneira, essas toxinas mostram uma atividade antiproliferativa12. Em adição, há estudos demonstrando que a toxina $B$ causa uma série de efeitos inespecíficos em células de mamíferos, tais como perda de potássio e cálcio intracelulares e diminuição na síntese de ácido ribonucléico e desoxirribonucléico com a conseqüente redução na síntese protéica 14 .

A literatura relata que a toxina $B$ é desprovida de efeitos enterotóxicos, ou seja, não é capaz de provocar secreção de fluidos, lesão morfológica, nem infiltrado inflamatório na mucosa intestinal, in vivo 27 . Por outro lado, Riegler et al36, demonstraram que essa toxina provoca, de forma dose-dependente, alterações eletrofisiológicas e morfológicas na mucosa colônica humana, montada em câmaras de Üssing36. Ademais, a toxina $B$ é cerca de mil vezes mais potente que a toxina $A$, no que diz respeito aos seus efeitos citotóxicos, para vários tipos de células de mamíferos em cultura3 26.

Recentemente, o nosso grupo de pesquisa demonstrou que as toxinas A e B do Clostridium difficile são potentes indutoras da migração de neutrófilos, em cavidade peritoneal e bolsa de ar subcutânea de ratos, dependentemente da presença de macrófagos 3741 . Ademais, foi evidenciado que ambas as toxinas são capazes de estimular macrófagos, in vitro, a liberar potentes mediadores inflamatórios, tais como, leucotrienos e citocinas 313741.

Essas evidências estão fundamentadas nos seguintes fatos: a) o aumento da população de macrófagos, pelo tratamento com tioglicolato, amplificou o efeito da migração de neutrófilos; $b$ ) a depleção de células peritoneais residentes, por meio da lavagem peritoneal, inibiu essa atividade; c) o tratamento dos animais com inibidores específicos de lipoxigenase, tais como MK 886 e BWA4C, também, bloqueou o referido evento; d) a incubação de soros anti-TNF- $\alpha$ e anti-IL-1 $\beta$ com toxina $A$ neutralizou a capacidade dessa toxina em promover infiltração de neutrófilos, em bolsas de ar subcutâneas. Evidenciou-se, ainda, que somente o soro anti-TNF- $\alpha$ inibiu essa atividade quando o estímulo inflamatório foi a toxina B; e e) Por fim, demonstrou-se que monocamadas de macrófagos estimulados com toxina $A$ ou $B$ foram capazes de liberar, no sobrenadante da cultura, fatores quimiotáticos para neutrófilos, in vivo31 3741.

Em adição, foi demonstrado que a toxina $A$ é capaz de estimular a síntese e a liberação de IL-1, por macrófagos em cultura, que, por sua vez, é capaz de induzir a proliferação de linfócitos 32 . Além do mais, Flegel et al13, evidenciaram que ambas as toxinas do $C$. difficile são potentes estimuladoras da liberação de TNF- $\alpha$, IL-1 e IL-6, por monócitos humanos, in vitro.

Está bem demonstrado em animais experimentais que a toxina $A$, através de seus efeitos enterotóxicos, é capaz de produzir uma intensa secreção de fluidos, aumento da permeabilidade intestinal e uma potente reação inflamatória aguda na mucosa intestinal, caracterizada por necrose epitelial, edema hemorrágico, ulceração e ativação de macrófagos e mastócitos, com a subseqüente mobilização de neutrófilos para o foco inflamatório7 212224272843 .

Corroborando esse fato, Lima et al24, demonstraram que a injeção de toxina $\mathrm{A}$ em alça intestinal de coelho, a partir de $2 \mathrm{~h}$, provoca uma significativa infiltração de células mononucleares e uma potente secreção intestinal semelhante àquela produzida pela toxina do Vibrio cholerae. Outros pesquisadores, desse mesmo grupo, evidenciaram ainda que esta secreção vem acompanhada por uma excessiva reação inflamatória, as quais foram bloqueadas por inibidores de fosfolipase $A_{2}$, de ciclooxigenase e por antagonistas do fator de agregação plaquetária 1115.

O resultado da inflamação intestinal provocada pela toxina $A$, no intestino de coelho, é um acentuado influxo de neutrófilos com a conseqüente destruição da mucosa intestinal7 2135 . Essa lesão celular é mais grave na extremidade da vilosidade, porém, toda a mucosa é envolvida por uma intensa reação inflamatória com áreas de necrose 35 . Ademais, por meio desse mesmo protocolo, observou-se que, diferentemente da toxina da cólera que provoca secreção de fluidos acelulares e ricos em proteínas, a toxina $A$ induz um potente efeito secretório intestinal caracterizado pela presença de exsudato que contém neutrófilos, monócitos e enterócitos descamados 3543 .

Os mecanismos envolvidos na resposta inflamatória da toxina A são complexos e envolvem, pelo menos em parte, a liberação de potentes mediadores inflamatórios, do tipo prostaglandina $\mathrm{E}_{2}$, e leucotrieno $\mathrm{B}_{4}$, leucotrieno 
$\mathrm{C}_{4}$, fator ativador de plaquetas, interleucina-1, interleucina-8, histamina e óxido nítrico, por células residentes da lâmina própria intestinal7 25293243.

Os mastócitos, da mesma forma que os macrófagos, têm sido implicados nas ações biológicas da toxina $A$. Nesse sentido, foi observado que a injeção desta toxina, na alça intestinal de coelho, induz um aumento na produção de histamina e $\mathrm{LTC}_{4}$ que são sintetizados especialmente por mastócitos 25. Observou-se, também, que o ketotifen (estabilizador de mastócitos) reduz a lesão, secreção de fluidos, permeabilidade intestinal e níveis de PAF, $\mathrm{LTC}_{4}$ e $\mathrm{LTB}_{4}$ provocados pela toxina $A$ no íleo de ratos 34 . Foi relatado, ainda, que essa toxina causa a liberação de uma protease específica, denominada RMCPII, por mastócitos da lâmina própria intestinal, que pode ser

pró-inflamatórios.

\section{REFERÊNCIAS BIBLIOGRÁFICAS}

1. Aktories K. Clostridial ADP-ribosylating toxins: Effects on ATP and GTP-binding proteins. Molecular and Cellular Biochemistry 138:167-176, 1994.

2. Anglim AM, Farr BM. Nosocomial diarrhea due to Clostridium difficile. Current Opinion in Infectious Diseases 7:602-608, 1994.

3. Baldacini O, Girardot R, Green GA, Rinn B, Monteil H. Comparative study of immunological properties and cytotoxic effects of Clostridium difficile toxin B and Clostridium sordellii toxin L. Toxicon 30:129-40, 1992.

4. Banno Y, Kobayashi T, Kono H, Watanabe K, Veno K, Nozawa Y. Biochemical characterization and biologic actions of two toxins (D-1 and D-2) from Clostridium difficile. Review of Infectious Diseases 6: S11-S20, 1984.

5. Barroso LA, Wang SZ, Phelps CJ, Johnson JL, Wilkins TD. Nucleotide sequence of Clostridium difficile toxin B gene. Nucleic Acid Research 18:4004, 1990.

6. Bartlett JG. Clostridium difficile: Clinical considerations. Review of Infectious Diseases 12:243-251, 1990.

7. Burakoff R, Zhao L, Celifarco AJ, Rose KL, Donovan V, Pothoulakis C, Percy WH. Effects of purified Clostridium difficile toxin A on rabbit distal colon. Gastroenterology 109:348-354, 1995.

8. Castagliulo I, LaMont JT, Letourneau R, Kelly C, O'keane JC, Jaffer A, Theoharides TC. Neuronal involvement in the intestinal effects of Clostridium difficile toxin A and Vibrio cholerae enterotoxin in rat ileum. Gastroenterology 107:657-665, 1994.

9. Dillon ST, Rubin EJ, Yakubovich M, Pothoulakis C, LaMont JT, Feig LA, Gilbert RJ. Involvement of Rasrelated $R$ ho proteins in the mechanisms of action of bloqueada pelo ketotifen 83033 34, reforçando, portanto, a participação dos mastócitos residentes da lâmina própria intestinal na enterotoxicidade da toxina A. Por outro lado, recentemente o nosso grupo demonstrou que essas células não participam do evento da migração de neutrófilos provocada pelas toxinas A e B37 41 .

Em resumo, fazendo-se uma análise global dos trabalhos ora discutidos, podemos concluir que os efeitos deletérios das toxinas $A$ e $B$ do Clostridium difficile sobre a mucosa intestinal, resultam de mecanismos indiretos mediados em parte por células inflamatórias, em especial macrófagos, mastócitos e neutrófilos, que, por sua vez, participam da fisiologia e imunofarmacologia do epitélio intestinal através da síntese e liberação de potentes mediadores

Clostridium difficile toxin A and B. Infection and Immunity 63:1421-1426, 1995.

10. Dove CH, Wang SZ, Price SB, Phelps CJ, Lyerly DM, Wilkins TD, Johnson JL. Molecular characterization of the Clostridium difficile toxin A gene. Infection and Immunity 58:480-488, 1990.

11. Fang G, Lima AAM, Thielman N, Fonteles $M$, Yotseff $P$, Lyerly D, Guerrant RL. Role of phospholipase A2 in the histologic, epithelial and secretory responses to Clostridium difficile toxin A. Biomedical Journal 1:1-5, 1994.

12. Fiorentini C, Thelestam M. Clostridium difficile toxin A and its effects on cells. Toxicon 29:543-567, 1991.

13. Flegel WA, Müller F, Daubener W, Fischer HG, Hadding $\mathrm{U}$, Northoff $\mathrm{H}$. Cytokine response by human monocytes to Clostridium difficile toxin A and toxin B. Infection and Immunity 59:3659-3666, 1991.

14. Florin I, Thelestam M. Polyphosphate-mediated protection from cellular intoxication with Clostridium difficile toxin $\mathrm{B}$. Biochimica et Biophysica Acta 85:131-136, 1984.

15. Fonteles MC, Fang G, Thielman NM, Yotseff PS, Guerrant $\mathrm{RL}$. Role of platelet activating factor in inflammatory and secretory effects of Clostridium difficile toxin A. Journal Lipid Mediators Cell Signalling 11:133-143, 1995.

16. George RH, Symonds JM, Dimock F, Brown JD, Arabi Y, Shinegawa N, Keighley MRB, Alexande-Williams J, Burdon DW. Identification of Clostridium difficile as a cause of pseudomembranous colitis. British Medical Journal 1:695, 1978.

17. Giry M, Popoff MR, Von Eichel-Streiber C, Boquet P. Transient expression of RhoA, B and C GTPases in HeLa cells potentiates resistance to Clostridium difficile toxins $\mathrm{A}$ and $\mathrm{B}$ but not to Clostridium sordellii lethal toxin. Infection and Immunity 63:4063-4071, 1995. 
18. Gröschel DH. Clostridium difficile infection. Critical Reviews in Clinical Laboratory Sciences 33:203-245, 1996.

19. Just I, Fritz G, Aktories K, Giry M, Popoff MR, Boquet P, Hegenbarth S, Von Eichel-Streiber C. Clostridium difficile toxin B acts on the GTP-binding protein Rho. The Journal of Biological Chemistry 269:10706-107012, 1994.

20. Just I, Selzer J, Von Eichel-Streiber C, Aktories K. The low molecular mass GTP-binding protein Rho is affected by toxin A from Clostridium difficile. The Journal of Clinical Investigation 95:1026-1031, 1995.

21. Kelly CP, Pothoulakis C, LaMont JT. Clostridium difficile colitis. The New England Journal of Medicine 330:257262, 1994.

22. Lima AAM, Innes DJ, Chadee K, Lyerly DM, Wilkins TD, Guerrant RL. Clostridium difficile toxin A interactions with mucus and early sequential histopathologic effects in rabbit small intestine. Laboratory Investigation 61:419425, 1989.

23. Lima AAM, Lima NL. Epidemiology, therapy and prevention of infection with Shigella organisms and Clostridium difficile. Current Opinion in Infectious Diseases 6:63-71, 1993.

24. Lima AAM, Lyerly DM, Wilkins TD, Innes DJ, Guerrant $\mathrm{RL}$. Effects of Clostridium difficile toxins $\mathrm{A}$ and $\mathrm{B}$ in rabbit small and large intestine in vivo and on cultured cells in vitro. Infection and Immunity 56:582-588, 1988.

25. Linevsky J, Becker S, Pothoulakis C, Holdridge M, Theoharides TC, LaMont JT. Clostridium difficile toxin A stimulates release of mast cell mediators in rabbit ileal loops. Gastroenterology 98:A459, 1990.

26. Lyerly DM, Krivan HC, Wilkins TD. Clostridium difficile: It's Disease and Toxins. Clinical Microbiology Reviews 1:1-18, 1988.

27. Lyerly DM, Lockwood DE, Richardson SH, Wilkins TD. Biological activities of toxins $A$ and $B$ of Clostridium difficile. Infection and Immunity 35:1147-1150, 1982.

28. Lyerly DM, Saum KE, MacDonad DK, Wilkins TD. Effects of Clostridium difficile toxins given intragastrically to animals. Infection and Immunity 47:349-352, 1985.

29. Mahida YR, Makh S, Hyde S, Gray T, Borriello SP. Effect of Clostridium difficile toxin $A$ on human intestinal epithelial cells: Induction of interleukin-8 production and apoptosis after cell detachment. Gut 38:337-347, 1996.

30. Mantyh CR, Pappas TN, Lapp JA, Washington MK, Neville LM, Ghilardi JR, Rogers SD, Mantyh PW, Vigna SR. Subtance $P$ activation of enteric neurons in response to intraluminal Clostridium difficile toxin $A$ in the rat ileum. Gastroenterology 111:1272-1280, 1996.

31. Melo Filho AA, Souza MHLP, Lyerly DM, Cunha FQ, Lima AAM, Ribeiro RA. Role of tumor necrosis factor and nitric oxide in the cytotoxic effects of Clostridium difficile toxin $A$ and toxin B on macrophages. Toxicon 35:743-752, 1997.
32. Miller PD, Pothoulakis C, Baeker TR, LaMont JT, Rothstein TL. Macrophage-dependent stimulation of $T$ cell-depleted spleen cells by Clostridium difficile toxin A and calcium ionophore 1. Cellular Immunology 126:153163, 1990.

33. Pothoulakis C, Castagliulo I, LaMont JT, Jaffer A, O'Keane JC, Snider RM, Leeman SE. CP-96,345, a substance $P$ antagonist, inhibits rat intestinal responses to Clostridium difficile toxin A but not cholera toxin. Proceedings of the National Academy of Sciences of the United States of America 91:947-951, 1994.

34. Pothoulakis C, Karmeli F, Kelly CP, Eliakim R, Joshi MA, O'keane CJ, Castagliulo I, LaMont JT, Rachmilewitz D. Ketotifen inhibits Clostridium difficile toxin A-induced enteritis in rat ileum. Gastroenterology 105:701-707, 1993.

35. Pothoulakis C, LaMont JT. Clostridium difficile colitis and diarrhea. Gastroenterology Clinics of North America 22:623-637, 1993.

36. Riegler M, Sedivy R, Pothoulakis C, Hamilton G, Zacherl J, Bischof G, Cosentini E, Feil W, Schiessel R, LaMont JT, Wenzl E. Clostridium difficile toxin B is more potent than toxin $A$ in damaging human colonic epithelium in vitro. The Journal of Clinical Investigation 95:2004-2011, 1995.

37. Rocha MFG, Maia MET, Bezerra LRPS, Lyerly DM, Guerrant RL, Ribeiro RA, Lima AAM. Clostridium difficile toxin $A$ induces the release of neutrophil chemotactic factors from rat peritoneal macrophages: Role of Interleukin-1 $\beta$, Tumor Necrosis Factor Alpha, and leukotrienes. Infection and Immunity 65:2740-2746, 1997.

38. Samore MH, Venkataraman L, Degirolami PC, Arbeit RD, Karchmer AW. Clinical and molecular epidemiology of sporadic and clustered cases of nosocomial Clostridium difficile diarrhea. American Journal of Medicine 100:3240, 1996.

39. Schmidt M, Rümenapp U, Bienek C, Keller J, Von EichelStreiber C, Jacobs $\mathrm{KH}$. Inhibition of receptor signaling to phospholipase D by Clostridium difficile toxin B. Role of Rho proteins. Journal of Biological Chemistry 271:24222426, 1996.

40. Sears CL, Kaper JB. Enteric bacterial toxins: mechanisms of action and linkage to intestinal Secretion. Microbiological Reviews 60:167-215, 1996.

41. Souza MHLP, Melo-Filho AA, Rocha MFG, Lyerly DM, Cunha FQ, Lima AAM, Ribeiro RA. The involvement of macrophage-derived tumor neccrosis factor and lipoxygenase products on the neutrophil recruitment induced by Clostridium difficile toxin B. Immunology 91:281-288, 1997.

42. Starr JM, Rogers TR, Impallomeni M. Hospital-acquired Clostridium difficile diarrhoea and herd immunity. The Lancet 349:426-428, 1997.

43. Triadafilopoulos G, Pothoulakis C, Weiss R, Giampaolo C, LaMont JT. Comparative study of Clostridium difficile 